\title{
Understanding straining induced changes in thermal properties of tropocollagen-hydroxyapatite interfacial configurations
}

\author{
Tao Qu and Vikas Tomar* \\ School of Aeronautics and Astronautics, \\ Purdue University, \\ West Lafayette, IN 47907, USA \\ Fax: (765)-494-0307 \\ Email: qut@purdue.edu \\ Email: tomar@purdue.edu \\ *Corresponding author
}

\begin{abstract}
The ability of a biomaterial to transport energy by conduction is best characterised in the steady state by its thermal conductivity and in the non-steady state by its thermal diffusivity. The complex hierarchical structure of most biomaterials makes the direct determination of the thermal diffusivity and thermal conductivity difficult using experimental methods. This study presents a classical molecular simulation-based approach for the thermal diffusivity and thermal conductivity prediction for a set of tropocollagen and hydroxyapatite-based idealised biomaterial interfaces. The thermal diffusivity and thermal conductivity are calculated using the presented approach at five levels of straining ( $10 \%$ compressive, $5 \%$ compressive, $0 \%, 5 \%$ tensile, $10 \%$ tensile) at $300 \mathrm{~K}$. The effects of straining, interfacial period and thickness of simulated systems on the thermal properties are analysed. Analyses point out important role played by interfaces and straining in determining biomaterial thermal properties including establishment of a notion that straining can be used to tailor the thermal properties (thermal diffusivity and thermal conductivity) of the organic-inorganic interfacial system and nanocomposite systems.
\end{abstract}

Keywords: tropocollagen; hydroxyapatite; thermal diffusivity; thermal conductivity; straining; interfacial configuration; molecular dynamics.

Reference to this paper should be made as follows: Qu, T. and Tomar, V. (2015) 'Understanding straining induced changes in thermal properties of tropocollagen-hydroxyapatite interfacial configurations', Int. J. Experimental and Computational Biomechanics, Vol. 3, No. 1, pp.62-81.

Biographical notes: $\mathrm{Tao} \mathrm{Qu}$ is currently a $\mathrm{PhD}$ candidate in the Interfacial Multiphysics Lab in the School of Aeronautics and Astronautics at Purdue University.

Vikas Tomar is an Associate Professor in the School of Aeronautics and Astronautics at Purdue University. He is the Director of the Interfacial Multiphysics Lab. He works in the general area of multiscale simulations and experimentation of bio-inspired and bio-enabled materials with an account of interface properties. 


\section{Introduction}

Hard biomaterials such as bone, dentin and nacre have primarily an organic phase [e.g., tropocollagen (TC)] and a mineral phase [e.g., hydroxyapatite (HAP) or aragonite] arranged in a staggered arrangement at the lowest fundamental structural length scale of $n m$.

Figure 1 (a) A schematic of the hierarchical structure of bone (b) A schematic showing of the atomistic constituents which form hydroxyapatite (hap) cell (c) A schematic of staggered and layered assembly of tropocollagen (TC) molecules (see online version for colours)

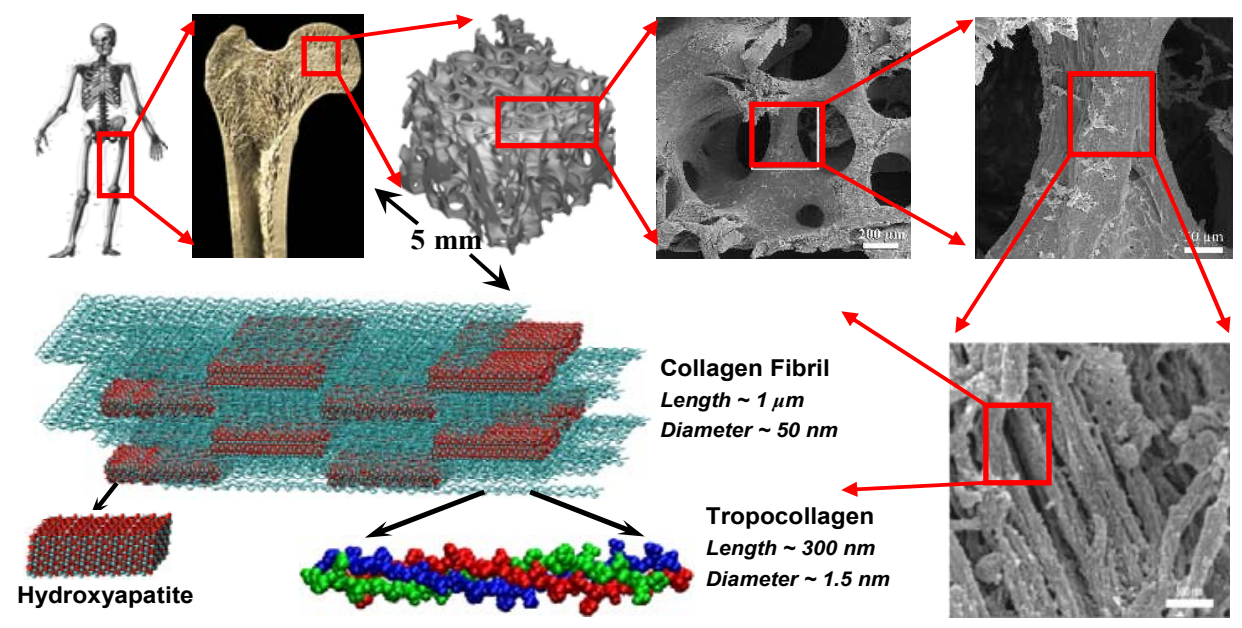

(a)

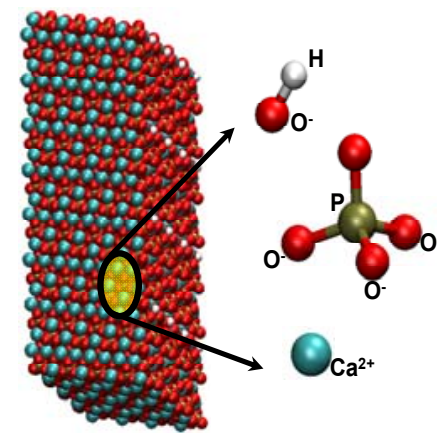

(c)

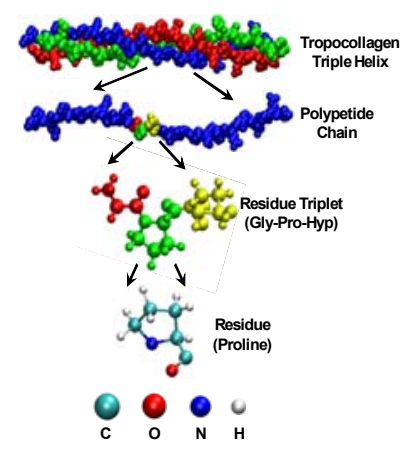

(d)

Note: Three different colours in TC molecule depict three polypeptide chains forming a triple helix

In the structural studies of such biological materials, it is observed that at the nanoscale, the mineral crystals are preferentially aligned along the length of the polypeptide molecules permitting maximum contact area in a staggered arrangement, (Fratzl et al., 1991; Landis et al., 1996a, 1996b; Weiner et al., 1983), (Figure 1). Biological materials usually have excellent multifunctional properties. For example, bone provides structural support as well as serves as home for blood cell production. Crustacean exoskeleton plays 
a set of functional roles including protection, support and sensing. The extraordinary adhesive capabilities of gravity defying gecko's foot have left researchers wondering for decades, (Autumn et al., 2000; Gao and Wang, 2005). Studies have shown that staggered hierarchical structure of interfaces in bone (TC-HAP interfaces) leads to its unique combination of toughness and strength properties. Not only the mechanical properties but also the thermal properties of bio-polymers are those of increasing interest recently. The latest studies on the deep-sea hydrothermal vent ecosystem found the deep-sea shrimp (Ramicaris exoculata) with the similar hierarchical structure of its amour-like exoskeleton has the capability of dealing with harsh and highly unstable thermal conditions (Cottin et al., 2010). However, how such an arrangement of interfaces contributes to physical properties of biological materials such as bone is not clear. Such an understanding is desired for real world applications that involve bone machining such as high speed drilling (Wiggins and Malkin, 1976), laser ablation (Nelson et al., 1988) and the curing of cements used in hip replacement (Huiskes, 1980). These applications require an understanding of heat propagation in bone and fundamental microstructural causes that affect heat propagation, particularly with focus on limiting the damage caused by heat. Such understanding can also point out important insights regarding the role of biological structural hierarchy in influencing physical properties. Biological principles, such as bio-heat transfer and biomimetic materials, are of increasing importance in the field of bio-medical engineering and multiple related disciplines, for example, prosthetic implants, regenerative medicine, self-healing materials and energy storage device. With this view this study presents a classical molecular simulation-based approach for the thermal diffusivity and thermal conductivity measurement in a set of TC-HAP-based idealised biomaterial interfaces.

One important aspect of understanding heat transfer mechanisms in biomaterials is the accurate knowledge of the organic-inorganic interfaces and its correlation with the thermal diffusivity and thermal conductivity of such biomaterials. This work investigates the effects of changes in HAP crystal shapes, interfacial periods, strain levels on the thermal properties of a set of idealised TC-HAP biomaterials using molecular dynamics (MD) simulations. The mechanical behaviour of biological materials with a view to understand the role of TC molecules and HAP mineral has been earlier analysed using experiments (Eppell et al., 2005; Gupta et al., 2006a, 2006b; Sasaki and Enyo, 1995; Sasaki and Odajima, 1996a, 1996b), modelling (Gao et al., 2003; Gao and Wang, 2005; Jager and Fratzl, 2000; Ji, 2008) and simulations (Buehler, 2006a, 2006b; Dubey and Tomar, 2009; 2010; Handgraaf and Zerbetto, 2006; Radmer and Klein, 2004; Zhang et al., 2007). Experimental techniques for investigating the thermal properties of the bone material as well as other biological materials have been reported over the years. Zelenov (1986) investigated the thermophysical properties of compact bone by heating the bone sample. Biyikli et al. (1986) measured experimentally the specific heat, thermal conductivity and thermal diffusivity of the human femora and the results agreed well with the data available at that time. Moses et al. (1995) introduced the inverse conduction technique, measured the thermal conductivity of cortical bone and compared the experimental results with literature values. However, such measurements have not shed light on the role of interfaces in determining physical properties. This is the focus of the present work.

MD-based analyses of the thermal behaviour for biomaterials are limited. The atomistic simulation has been performed on $\mathrm{Si}, \mathrm{Ge}$ and other semiconductor materials and proved to be the efficient method to analyse the thermal behaviour of solids under the 
conditions where the experiment is difficult to perform. Samvedi and Tomar (2009, 2010, 2011) analysed the thermal behaviour of Si-Ge biomimetic nanocomposite as a function of strain and structural arrangement at a range of temperatures using non-equilibrium molecular dynamics (NEMD) simulations. Jeng et al. (2008) have used Monte Carlo simulations to study the phonon transport and thermal conductivity reduction in the biomimetic nanocomposites with Si nanoparticle embedded in Ge host. Similar Monte Carlo analyses for the nanocomposites with Si tubular nanowires in Ge host have been performed by Yang et al. (2005). In a recent work, Huang et al. (2009) have used MD simulations to analyse similar systems as analysed by Yang et al. (2005). Yang and Chen (2004) have theoretically studied phonon thermal conductivity of periodic two dimensional nanocomposites with nanowires embedded in a host semiconductor material using phonon Boltzmann equation. Algaer and Müller-Plathe (2011) reviewed and discussed known methods and their accuracy for calculating the thermal conductivity. Almost all analyses have focused on measuring thermal conductivity which characterises the ability of material to transport energy in the steady state. However, for biomaterial, the thermal diffusivity, which characterises the energy transportation in the non-steady state, is of significant importance due to reasons outlines earlier. Qu and Tomar (2014) recently analysed the thermal diffusivity and conductivity of TC-HAP interfaces with an account of the temperature and interfacial arrangement. This investigation presents an analysis of the thermal properties of TC-HAP interfacial material systems with different structural configurations and strain levels with the method reported by $\mathrm{Qu}$ and Tomar (2014) using NAMD simulation package. Emphasis is on understanding of the thermal behaviour of a set of TC-HAP biomaterials using equilibrium molecular dynamics (EMD) simulations and NEMD simulations at different strain levels. NEMD simulations are performed to calculate the thermal diffusivity values and EMD simulations are performed to calculate the specific heat values. Results from both sets of simulations are then used to calculate thermal conductivity values.

\section{Method and framework}

There are two approaches to analyse thermal transport using MD, (Schelling et al., 2002):

1 an EMD-based approach that uses fluctuation dissipation theorem or the Green-Kubo method

2 a NEMD approach that is based on imposing a heat flux and subsequently measuring thermal diffusivity using the Fourier equation of thermal conduction.

NEMD-based approach has been found most suitable in the case of heterogeneous systems. Samvedi and Tomar $(2009,2010,2011)$ have used such an approach for a variety of nanocomposite simulations. The emphasis in this work is on the analysis of the effects of component thickness, interfacial period and levels of straining on the thermal diffusivity and thermal conductivity of hierarchical TC-HAP interfacial systems.

\subsection{EMD simulation to calculate the specific heat}

For the EMD simulations, the structure is equilibrated in canonical (NVT) ensemble. The specific heat (heat capacity per unit volume) can be calculated as 


$$
C_{V}=(\partial E / \partial T)_{V}=\left\langle\delta E^{2}\right\rangle / k_{B} T^{2},
$$

where $E$ is the total energy of the simulation system, including conformational energy (bond, angle, dihedral, improper) and non-bonded energy (VDW, electrostatic) (Isgro et al., 2012), $k_{B}$ is the Boltzmann constant and $T$ is the simulation temperature. $\left\langle\delta E^{2}\right\rangle$ is the magnitude of the energy fluctuations in the NVT ensemble, which can be expressed as

$$
\left\langle\delta E^{2}\right\rangle=\left\langle E^{2}\right\rangle-\langle E\rangle^{2}
$$

where $\left\langle E^{2}\right\rangle$ and $\langle E\rangle$ are thermodynamic averages. The thermodynamic average of an arbitrary quantity $A$ can be expressed as

$$
\langle A\rangle=\frac{1}{N} \sum_{i=1}^{N} A\left(t_{i}\right),
$$

and

$$
\langle\delta A\rangle \approx \frac{1}{N} \sum_{i=1}^{N}\left[A\left(t_{i}\right)-\langle A\rangle\right],
$$

where $i$ is the MD simulation time series, $N$ is the total time steps of MD simulation.

\subsection{NEMD simulation to calculate the thermal diffusivity}

For NEMD simulations, a thermal gradient is established by imposing $T_{\text {hot }}=T+50 \mathrm{~K}$ in the hot reservoirs (shown in Figure 2) and the computational system is equilibrated for 50,000 time steps. This computational method is developed based on the non-equilibrium simulation method for heat diffusion in NAMD simulation package (Phillips et al., 2005) and was reported in the earlier study (Qu and Tomar, 2014).

Figure 2 A schematic showing the NEMD simulation setup (see online version for colours)

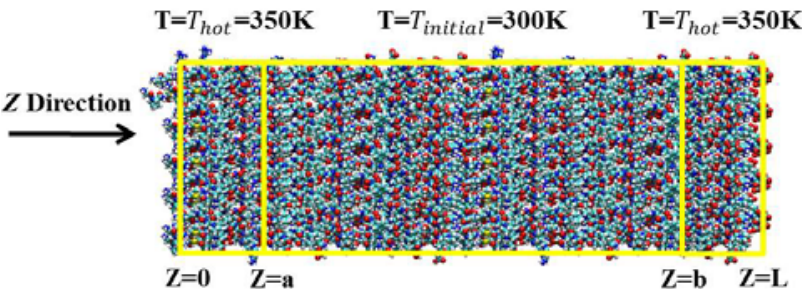

Note: Hot reservoirs are the parts from $Z=0$ to $Z=a$ and from $Z=b$ to $Z=l$ in $Z$ direction

During the non-steady state, the temperature $T(z, t)$ of the computational system is governed by the heat equation

$$
\frac{\partial T(z, t)}{\partial t}=D\left(\frac{\partial^{2} T(z, t)}{\partial z^{2}}\right),
$$


where $D$ is the thermal diffusivity. Based on the finite difference method, the values of $D$ are calculated using equation (6) for each time step as,

$$
\frac{T_{i}^{k+1}-T_{i}^{k}}{\Delta t}=D \frac{T_{i-1}^{k}-2 T_{i}^{k}+T_{i+1}^{k}}{h^{2}}
$$

where $T_{i}^{k}=T\left(z_{i}, t^{k}\right)$, and $z_{i}=i h, i=0,1, \ldots m, h=\frac{\text { Length }}{m}, t^{k}=k \Delta t, k=0,1 \ldots n, \Delta t=$

Time/n. Temperature is related to the kinetic energy $K$ of the computational system by

$$
K=\left\langle\frac{1}{2} m v^{2}\right\rangle=\frac{3}{2} k_{B} T
$$

$T_{i}^{k}$ is obtained by dividing the computational system into several slabs in $\mathrm{z}$ direction. Thermal diffusivity values are calculated as a function of total simulation time. Different sets of computation of diffusivities are performed by dividing the non-steady state time period into different number of time steps, i.e., different values of $\Delta t$ is used for different sets of computation. Cumulative moving average of diffusivities is calculated with the increasing of $\Delta t$, plotted as the red dots in Figure 5. Calculations were stopped once the convergence in the computed $D$ values is obtained, indicated by the blue curve in Figure 5. The thermal conductivity value $k$ can be calculated using the relation (8) based on the known specific heat value calculated from the EMD simulation and the obtained thermal diffusivity as

$$
k=\rho C_{V} D,
$$

where $\rho$ is the density of the computational system.

\subsection{Simulation setup}

Three dimensional (3-D) MD simulations are performed on different types of TC-HAP interfacial systems (Figure 3): TC molecular systems and HAP molecular systems with various thicknesses, TC-HAP interfacial systems with different number of interfacial periods, denoted as (TC-HAP $)_{2},(\text { TC-HAP })_{3}$ and (TC-HAP $)_{4}$ and the structurally distinct TC-HAP interfacial supercells one with HAP crystals in needle shape (NS) and the other with HAP crystals in plate shape (PS). In nanocomposite supercells, TC molecules are aligned in a direction parallel to the c-axis (longitudinal axis) of HAP crystals. The supercells are generated based on the information on tension-shear type of load transfer mechanism possible for staggered arrangement shown in Figure 1, (Gao, 2006; Ji, 2008). The size of the supercells was chosen as the maximum size which could be computationally analysed using 3-D MD in a period of at most 15 days (on Dell PowerEdge SC1435 server, $2 \times$ Dual-core AMD Opteron Model 2218 (2.6 GHz) processors and approximately 32 processors). 
Figure 3 A schematic showing of the system being analysed (a) HAP systems with different thickness (b) TC molecules and TC-HAP interfacial structures (longitudinal TC molecules) (c) TC-HAP interfacial structures (transverse TC molecules) with different number of interfacial periods (d) TC-HAP nanocomposite with needle shaped HAP molecules and TC-HAP nanocomposite with plate shaped hap molecules (see online version for colours)

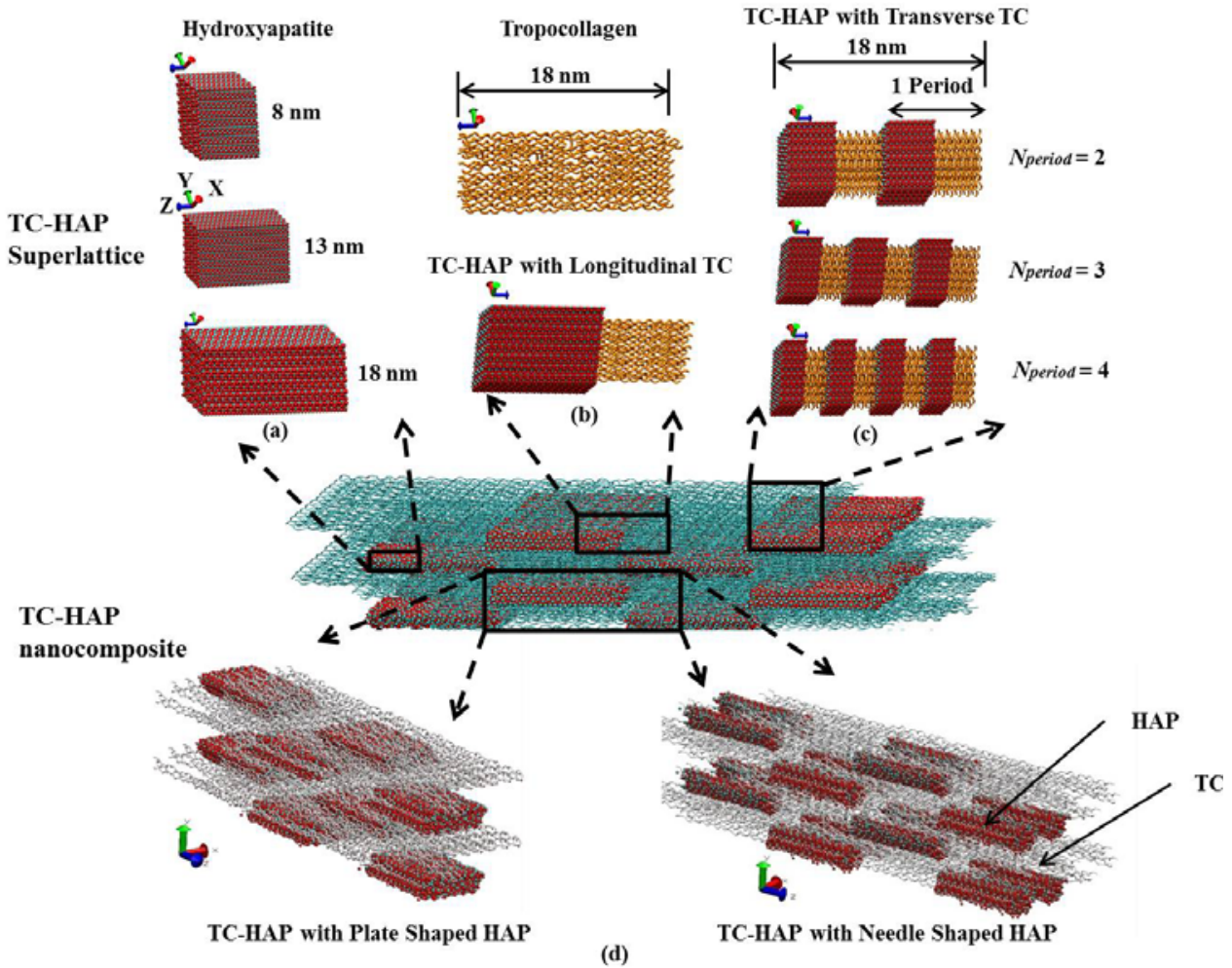

The NAMD package, (Phillips et al., 2005), is used to perform MD simulations. Due to computational infeasibility of performing atomistic analysis of supercells with full length TC molecules, only a segment of TC full length molecule is used in the supercells. The segment length chosen is the same as used by other researchers in past, (Bella et al., 1994; Buehler, 2006a, 2006b; Klein and Huang, 1999; Kramer et al., 2000; Lorenzo and Caffarena, 2005; Vesentini et al., 2005). The full length TC molecule [shown in Figure 1(b)] residue sequence as submitted in reference (Orgel et al., 2006) (pdb id ' 1 YGV') is used to generate the atomic level structure of the TC molecule using the gencollagen package, (Huang et al., 1998). Hydrogen atoms are added by 'psfgen' module of NAMD package. The HAP supercell [shown in Figure 1(c)] is generated by using fractional coordinate and unit cell parameter information given in reference (Elliott et al., 1973), for monoclinic phase of HAP with space group $\mathrm{P} 2_{1} / \mathrm{b}, \mathrm{a}=9.4214 \AA \mathrm{A}, \mathrm{b}=2 \mathrm{a}$, $\mathrm{c}=6.8814 \AA$ and $\gamma=120^{\circ}$. The HAP supercell consists of 64 unit cells (4 unit cells along all three crystallographic axes) with 88 atoms per unit cell and one TC molecule consists of 1135 atoms. The sizes of the supercells PS and NS were chosen based on two constraints: 
1 the maximum size which could be computationally analysed using 3-D MD in a period of almost 15 days

2 the interfacial supercell should be such that TC and HAP could be accommodated in a rectangular geometry.

A combination of CHARMM22 force field, (Ponder and Case, 2003) and an inorganic force field reported earlier for HAP, (Bhowmik et al., 2007; Hauptmann et al., 2003), is used to model the atomistic interactions. Geometric mean is used for van der Waals parameters for defining interactions at the TC-HAP interface. Particle mesh Ewald method is used to calculate electrostatic interactions with a cut off of $12 \AA$. The CHARMM22 force field and topology parameters for TC molecule are directly obtained from CHARMM website, (Ponder and Case, 2003). Parameters for a non-standard residue, hydroxyproline (HYP), are obtained using the procedure outlined by Anderson (2005). All simulations are performed using NAMD package as it works better with large bio-molecules and can be scaled well to very large processor counts (Phillips et al., 2005). The simulations are performed with periodic boundary conditions (PBC) imposed on the supercells. For NEMD simulations, the temperature gradients are applied using the 'TCOUPLE' option with the thermal gradient of $50 \mathrm{~K}$ in NAMD (Phillips et al., 2005). Simulations are performed on systems at five different strain levels $(5 \%, 10 \%$ compressive and $5 \%, 10 \%$ tensile, $0 \%$ ) for 50000 time steps ( $50 \mathrm{ps}$ ) at $300 \mathrm{~K}$. The strain is applied in the $z$ direction by stretching (or compressing) with a strain increment of $\varepsilon=$ $5 \%$ (or $\varepsilon=-5 \%$ ) in the until $10 \%$ (or $-10 \%$ ) strain level is reached. In order to account for Poisson's ratio $(v)$ effect, the supercell is compressed (or stretched) by $v \varepsilon \%$ in the transverse directions. A fixed value of $v(=0.3$ based on average of the reported literature values for TC-based materials) is used only initially. Afterwards, the supercell is equilibrated and actual Poisson's ratio for the supercell automatically arises out of transverse atomic displacements during supercell equilibration. Velocities of atoms are output every 10 time steps. Velocity profile of $10 \mathrm{ps}$ of the entire simulation from $300 \mathrm{~K}$ is extracted for the thermal diffusivity computation. For EMD simulations, NVT ensemble is applied and systems with the applied strain (5\%,10\% compressive and 5\%, $10 \%$ tensile, $0 \%)$ are equilibrated for 100000 time steps (100 ps) at $300 \mathrm{~K}$ without thermal gradient. The strain is applied in the $z$ direction. Energies for systems are output every tem time steps.

\section{Results analyses and discussion}

Figures 4(a) and (b) display the temperature profile of the pure TC system at the temperature of $300 \mathrm{~K}$ with the strain level of $0 \%$. Figure 4(a) describes the temperature as the function of time for the entire simulated system with hot reservoirs (from 0 to $L$ in $z$ direction shown in Figure 2). Figure 4(b) describes the temperature as the function of time for the simulated systems without hot reservoirs (from $a$ to $b$ in $z$ direction in Figure 2). Figure 4(c) displays the temperature profile of the simulated system with hot reservoirs (from 0 to $L$ in $z$ direction shown in Figure 2) at non-steady state (the time scales on the $\mathrm{x}$-axis indicate the $10 \mathrm{ps}$ time period of the unsteady heat diffusion state which is not corresponding to the time scales of the entire simulation, Figure 4(a) which is used to calculate the thermal diffusivity values at the temperature of $300 \mathrm{~K}$. 
Figure 4 (a) The temperature profile of a trial system as $z$ varies from 0 to $L$ (Figure 2) (b) The temperature profile as $z$ varies from $a$ to $b$ (Figure 2) (c) Temperature profile as $z$ from 0 to $L$ (Figure 2 ) at the non-steady state (see online version for colours)
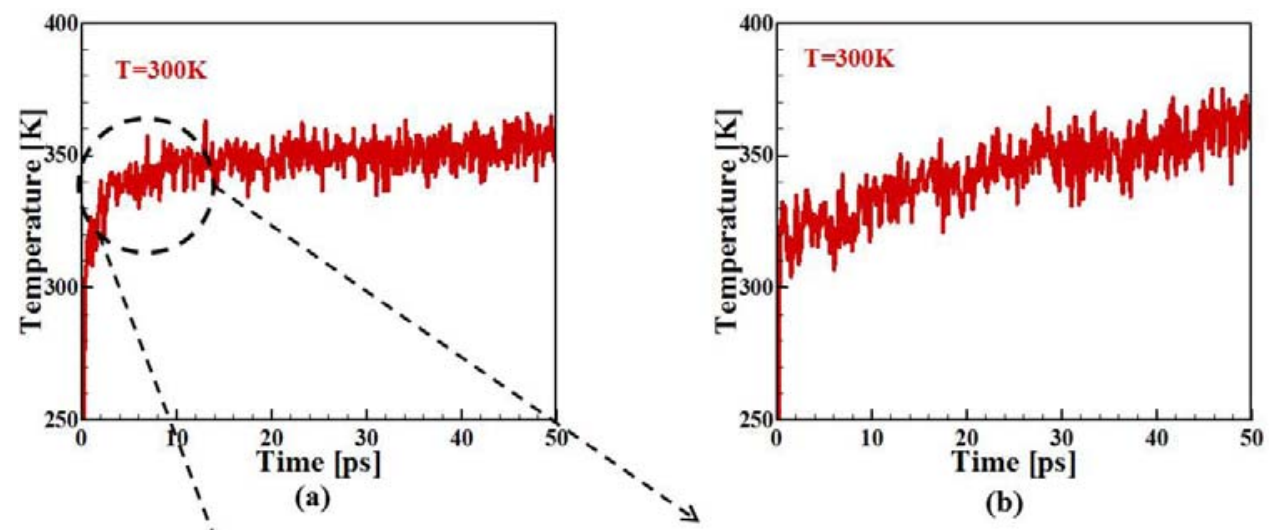

(b)

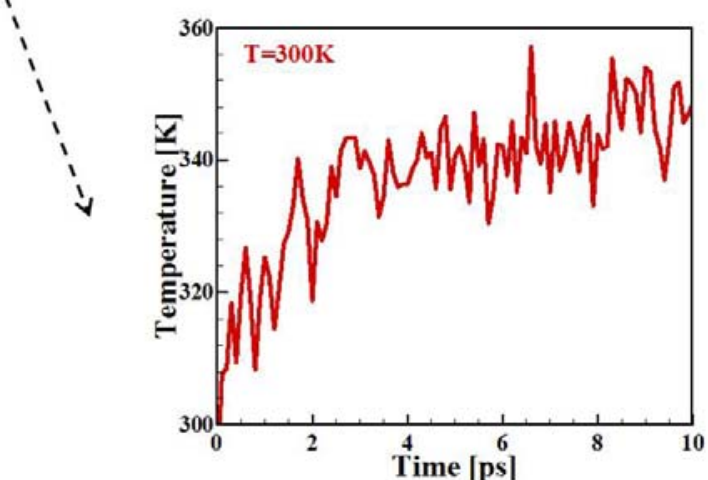

(c)

The thermal diffusivity of the simulated systems was calculated using the method described in Section 2.2. Figure 5 shows the convergence obtained in thermal diffusivity values as a function of simulation time for a TC system. Other systems showed similar characteristics in convergence. Calculations were stopped once the convergence in the computed $D$ values is obtained. The calculated thermal diffusivity varies from $1 \times 10^{-7}$ $\mathrm{m}^{2} / \mathrm{s}$ to $7 \times 10^{-7} \mathrm{~m}^{2} / \mathrm{s}$ of the simulated systems at different strain levels at the temperature of $300 \mathrm{~K}$. The reported values of thermal diffusivity (Biyikli et al., 1986; Zelenov, 1986) using experimental measurements are varying from $1 \times 10^{-7} \mathrm{~m}^{2} / \mathrm{s}$ to $70 \times 10^{-7} \mathrm{~m}^{2} / \mathrm{s}$ of bone samples at a temperature range of $20^{\circ} \mathrm{C}$ to $100^{\circ} \mathrm{C}$. The thermal conductivity of the system was calculated using the equation (8) with the known diffusivity values and the specific heat calculated from EMD simulation. The calculated thermal conductivity varies from $0.3 \mathrm{~W} / \mathrm{m} / \mathrm{K}$ to $14 \mathrm{~W} / \mathrm{m} / \mathrm{K}$ of the simulated systems at different strain levels at the temperature of $300 \mathrm{~K}$. The reported values of thermal conductivity (Biyikli et al., 1986; Zelenov, 1986) using experimental measurements are varying from $0.2 \mathrm{~W} / \mathrm{m} / \mathrm{K}$ to $13 \mathrm{~W} / \mathrm{m} / \mathrm{K}$ of bone samples at a temperature range of $20^{\circ} \mathrm{C}$ to $100^{\circ} \mathrm{C}$. The denaturation of collagen is responsible for the change of the specific heat of the system and the rapid variation of the specific heat of collagen is observed in the range of temperature of 320 340 K (Nadareishvili, 2012; Tiktopulo and Kajava, 1998). The significant effect of 
the temperature on the thermal properties of TC molecules has been studied earlier $(\mathrm{Qu}$ and Tomar, 2014). The specific heat was computed for each system equilibrated at $300 \mathrm{~K}$ in current study, thus collagen denaturation does not affect much on the properties reported in this study.

Figure 5 Convergence of the thermal diffusivity values as the function of the simulation time (see online version for colours)

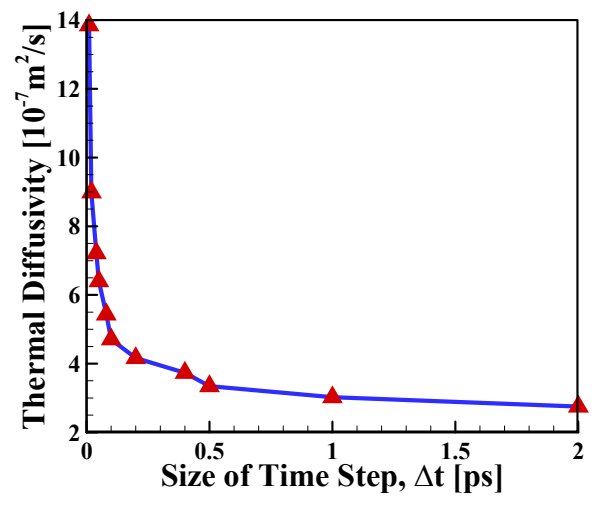

Figure 6 (a) Temperature profile during the unsteady heat diffusion (b) Thermal diffusivities (c) Specific heat values (d) Thermal conductivity values of HAP systems as a function of the thickness of the system (see online version for colours)

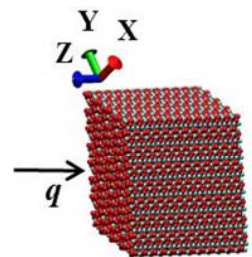

$L=8 \mathrm{~nm}$

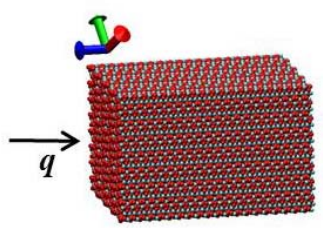

$L=13 \mathrm{~nm}$

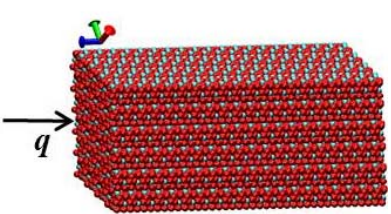

$L=18 \mathrm{~nm}$

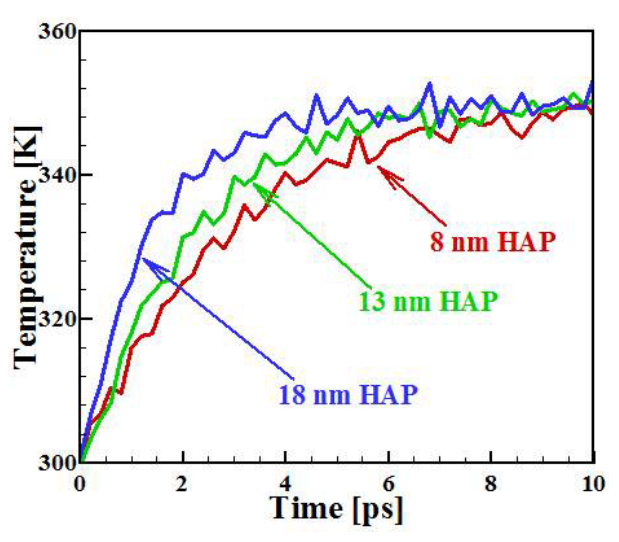

(a)

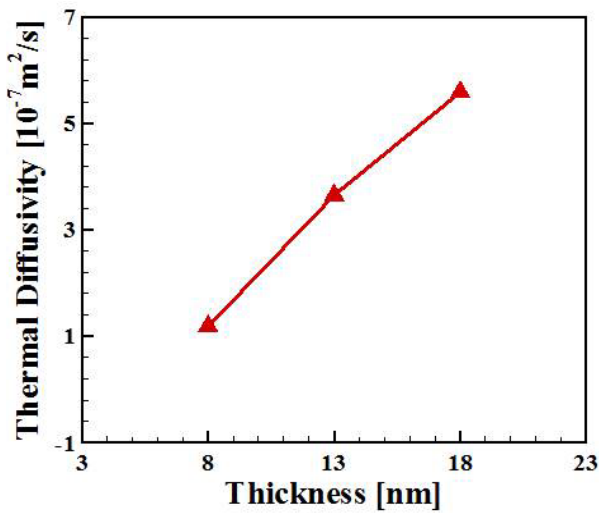

(b) 
Figure 6 (a) Temperature profile during the unsteady heat diffusion (b) Thermal diffusivities (c) Specific heat values (d) Thermal conductivity values of HAP systems as a function of the thickness of the system (continued) (see online version for colours)

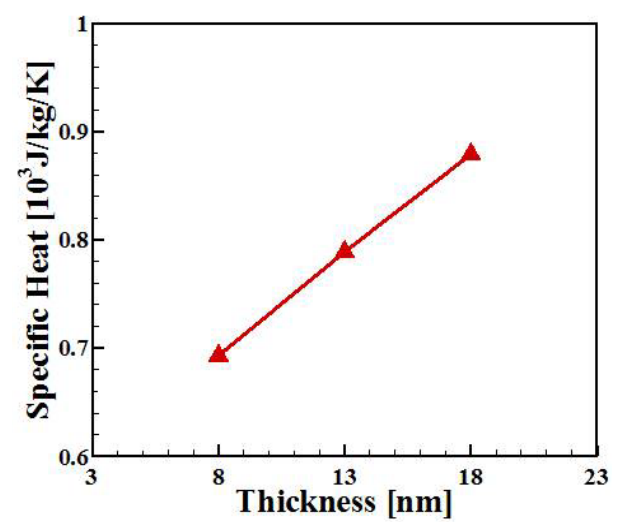

(c)

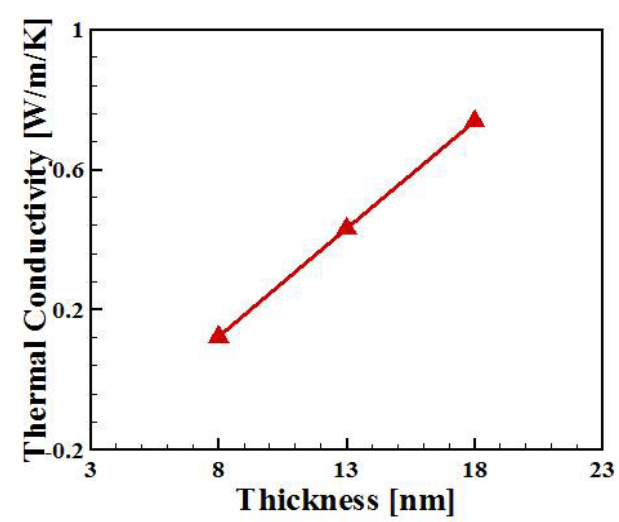

(d)

\subsection{Effect of thickness of the systems}

Figures 6(a) to 6(d) display the temperature profile during the unsteady heat diffusion and the correspondingly calculated thermal properties of the shown HAP systems with different thicknesses. As shown in Figures 6(a), the temperature increases faster (achieves steady state faster) in the case of the thicker HAP systems (the slope of the temperature profile curve $k_{\text {blue }}>k_{\text {green }}>k_{\text {red }}$ ), which leads to higher thermal diffusivity for the systems with higher thickness as shown in Figure 6(b). In order to thoroughly investigate the thickness effect on the thermal diffusivities of the HAP material systems, the specific heat and the thermal conductivity values were also calculated. Specific heat was calculated by averaging the total energy of the systems at the equilibrium state. As shown, there is a significant size effect present. The systems with higher thickness have higher specific heat values, Figure 6(c). Thermal conductivity values also increase as the thickness of the systems increases. This trend can be explained by a drop in the overall temperature gradient owing to increase in the overall length Samvedi and Tomar (2009, $2010,2011)$. The size effect can also be explained based on the expression of the thermal conductivity as given by kinetic theory of gases, equation (9).

$$
k=\frac{1}{3} C v l \text {. }
$$

where $C$ is the specific heat, $v$ is the velocity of phonons and $l$ is the mean free path (MFP) of the phonons. Since the simulations are performed at sub-nanometer length scale, the MFP corresponds to the thin film thickness in this case (Samvedi and Tomar, $2009,2010,2011)$. With the increasing of the thickness of the system, the phonon group velocity does not change at the same temperature. However, the specific heat increases as shown in Figure 6(c) and the MFP increases as the length increases, which leads to the increase in the thermal conductivity with increasing HAP thickness. Overall effect of the trends in thermal conductivity and specific heat is an increase in thermal diffusivity with increase in thickness. 
Figure 7 (a) Temperature profile during the unsteady heat diffusion (b) Thermal diffusivities (c) Specific heat values (d) Thermal conductivity values of HAP systems as a function of the TC-HAP systems with different number of interfacial periods (see online version for colours)

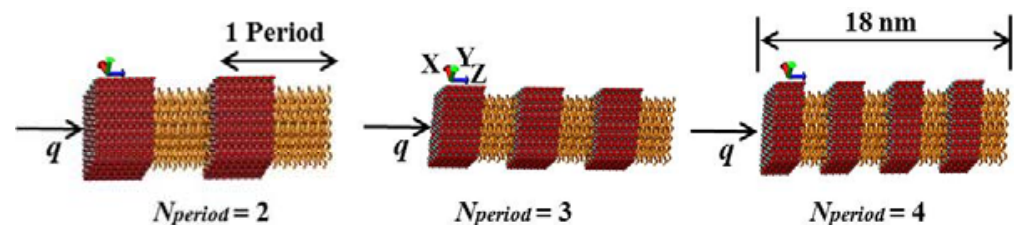

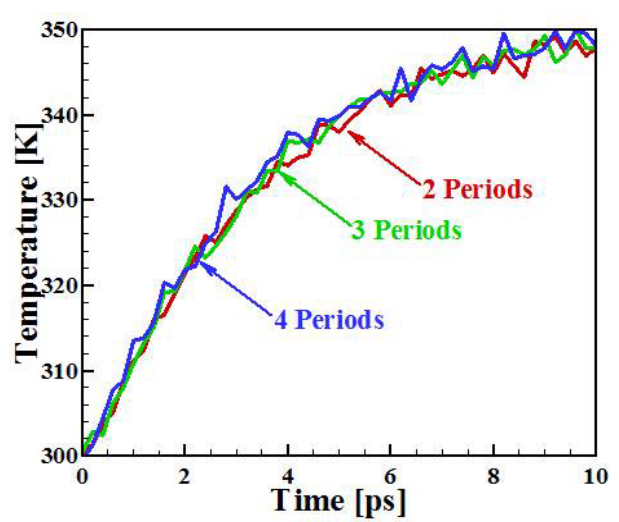

(a)

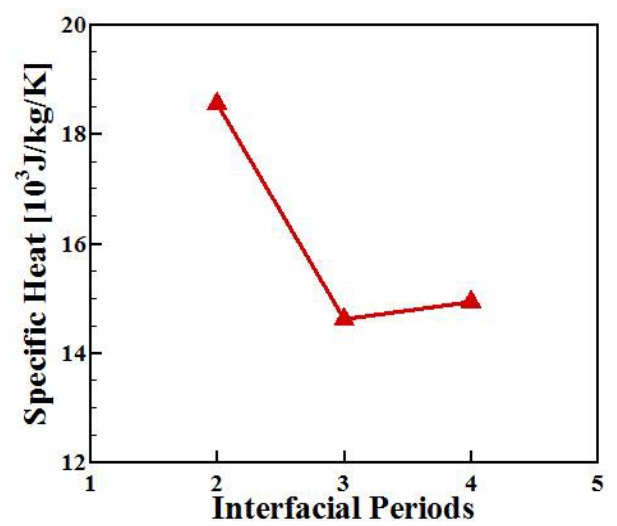

(c)

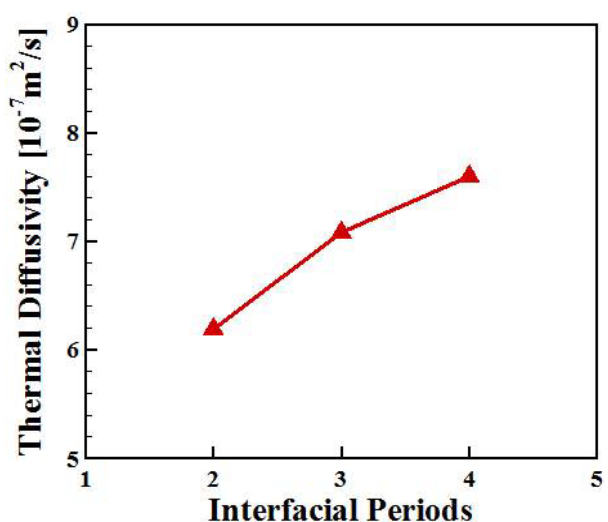

(b)

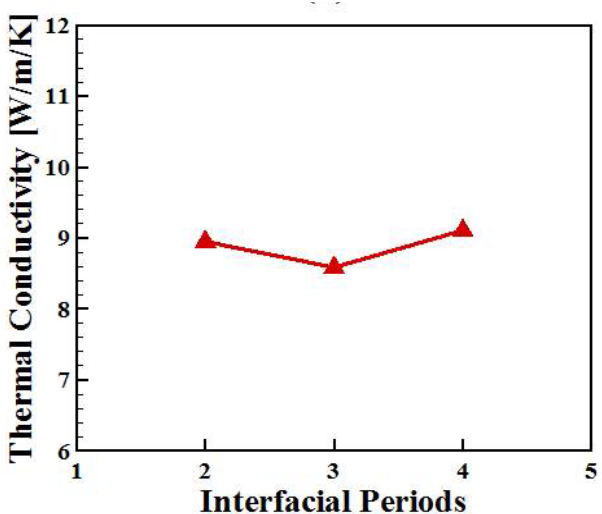

(d)

\subsection{Influence of number of interfacial periods}

The nanocomposite structure of bone is formed by the osteoblast that creates the HAP crystals subsequently both intrafibrillar and extrafibrillar to secret a collagenous extracellular matrix (Boonrungsiman et al., 2012). To investigate the structures in bone other than the mineral covering collagen fibrils, demineralisation is necessary (Wallace, 2012). Since the entire fibril is not exposed because of the mineralisation/ demineralisation processes, it is essential to understand the thermal mechanical properties of the collagen fibrils with different amount of mineralisation/demineralisation 
(Boonrungsiman et al., 2012; Wallace, 2012). The TC-HAP interfacial structures with different number of interfacial periods investigated in this study can simulate this situation. Figures 7 (a) to $7(\mathrm{~d})$ display the temperature profile and the thermal properties of the TC-HAP interfacial structures with the same overall length but different number of interfacial periods, denoted as (TC-HAP $)_{2}$, (TC-HAP $)_{3}$ and (TC-HAP) $)_{4}$. As shown in Figure 7(a), the temperature change as a function of time for all three kinds of systems has a slight difference in the slope (i.e., $k_{\text {blue }}>k_{\text {green }}>k_{\text {red }}$ ), which results in a slight increase (i.e., from $6 \times 10^{-7} \mathrm{~m}^{2} / \mathrm{s}$ to $8 \times 10^{-7} \mathrm{~m}^{2} / \mathrm{s}$ ) in thermal diffusivity with increasing the number of interfacial periods of the systems, Figure 7(b). In materials with predominantly covalent bonds (i.e., TC molecules), the total energy comes from the non-bonded interactions (i.e., electrostatic and van der Waals forces) and the covalent chemical bonding (i.e., bond, angle, dihedral, improper). The contribution of covalent bonds is significantly higher. Adding interfaces by introducing HAP molecules results in reduction of the contribution made to total system energy by the covalent chemical bonds. The reason is that introduction of interface leads to the interfacial bonding energy mainly coming from the electrostatic and van der Waals forces. Therefore, increasing the number of interfacial periods leads to the reduction of the total energy of the system which results in the decrease of energy required to induce thermal motions that leads to temperature increase. This directly results in decrease of the specific heat values as a function of increase in number of periods, Figure 7(c). Increase in interfacial period from three to four does not change specific heat since the ratio of interface area to the total TC part volume effectively remains same for three and four interfacial periods (since overall length is constant).

The change in thermal conductivity as a function of change in the number of interfacial periods is affected by several factors. Increasing the number of interfacial periods leads to higher cumulative thermal boundary resistance to the heat flow leading to reduction in thermal conductivity. In addition, the system with less number of interfaces also has thicker monolayer films (since overall length is constant) leading to increase in thermal conductivity. The contribution of cumulative effect is that thermal conductivity remains almost constant as a function of increase in number of interfacial periods, (Samvedi and Tomar, 2009), Figures 7(d). The change of the specific heat and thermal conductivity discussed above and the relation of the diffusivity, specific heat and conductivity expressed by equation (8) lead to the change of the thermal diffusivity with increasing the number of the interfacial periods, i.e., thermal diffusivity increasing as a function of increase in number of interfacial periods, Figures 7(b).

\subsection{Influence of straining}

Figures $8(\mathrm{a})$ to $8(\mathrm{c})$ display thermal properties of TC, HAP and a TC-HAP interfacial system as a function of strain. As shown, straining has much more significant effect on the thermal properties of the TC systems than that on the HAP systems. It is observed that the rate of change of thermal diffusivity, specific heat and thermal conductivity of the examined HAP system is much smaller than that of TC system, Figures 8(a), 8(b) and 8(c). This is attributed to the difference between the crystalline structure of the HAP and the polymer chain structure of TC. Therefore, in TC system, the straining would have more significant effect on the bond stretching, bond bending and bond torsion potentials, which leads to the significantly variation of the specific heat as well as the other 
properties as a function of strain. As shown, specific heat and thermal conductivity of the interface system lies in between that of TC and HAP. However, due to differences in chemical bonding involved between HAP and TC, the thermal properties of interface are not exactly in between those of TC and HAP. Interface is observed to be an important factor which can prove significant in tailoring the thermal mechanical properties of bio material systems. The specific heat and thermal conductivity values of the TC and HAP material system with the strain level of $10 \%$ were not calculated as strains at that level led to slip in the case of TC and fracture in the case of HAP. The fracture strain of HAP system is consistent with the results reported in the earlier study (Dubey and Tomar, 2009), which is different from the fracture strain experimentally measured for bone at the macro-scale. This can be attributed to the difference in length scale and the porosity effects (Dubey and Tomar, 2009) on the macro-scale bone samples. However, the TC-HAP interfacial system does not undergo similar slip or fracture at $10 \%$ strain because of the interfacial bonding between the TC fibrils and the HAP mineral. Therefore, introducing the inorganic-organic interfaces improves the performance of the thermal as well as the mechanical behaviour of the materials.

Figure 8 (a) Thermal diffusivities (b) Specific heat values (c) Thermal conductivity values of a TC, HAP and a TC-HAP interfacial system as a function of strain (see online version for colours)

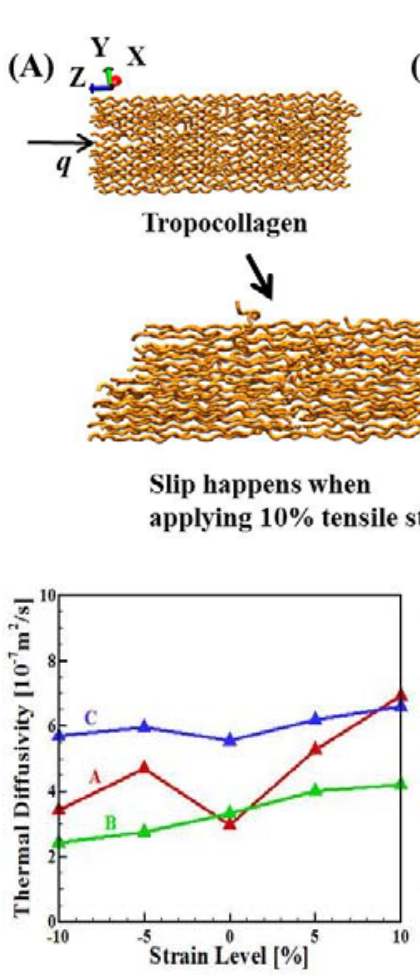

(a)

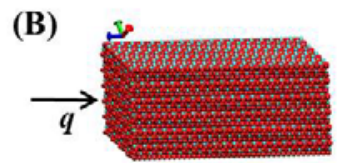

Hydroxyapatite

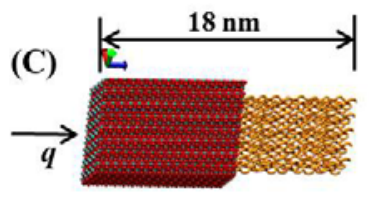

TC-HAP with Longitudinal TC

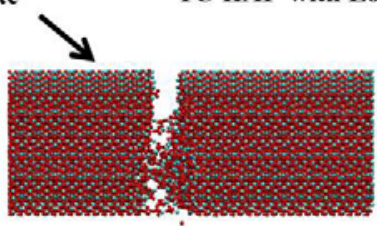

Fracture happens when applying $10 \%$ tensile strain

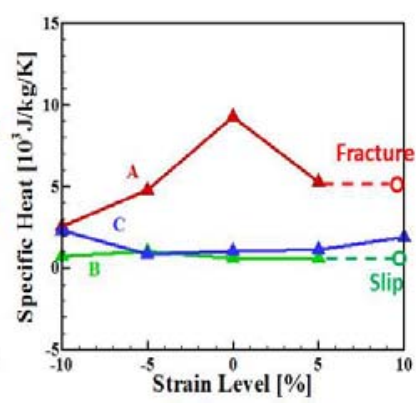

(b)

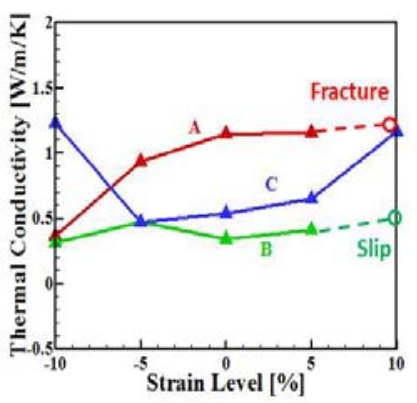

(c) 
Figure 9 (a) Thermal diffusivities (b) Specific heat values (c) Thermal conductivity values of two TC-HAP nanocomposite systems as a function of strain (see online version for colours)

(A)

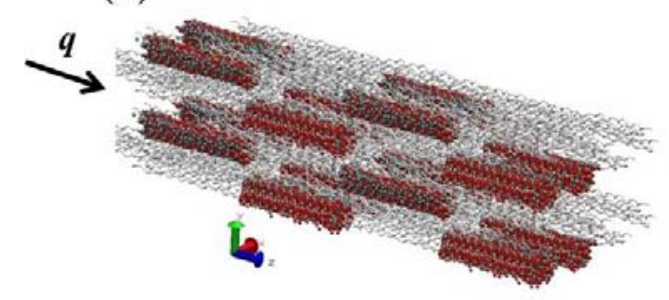

Needle Shaped
(B)

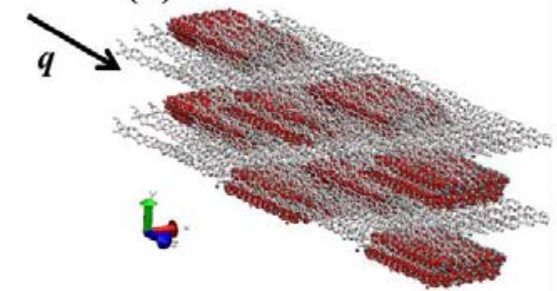

Plate Shaped

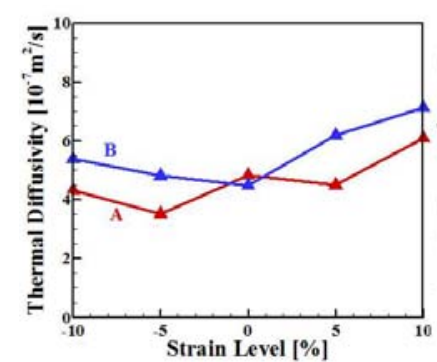

(a)

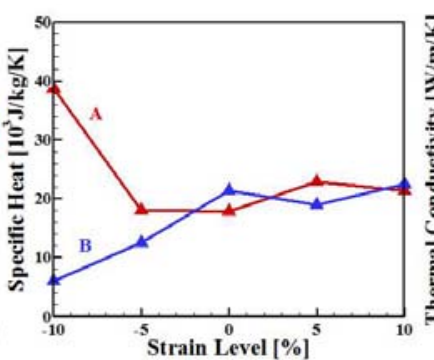

(b)

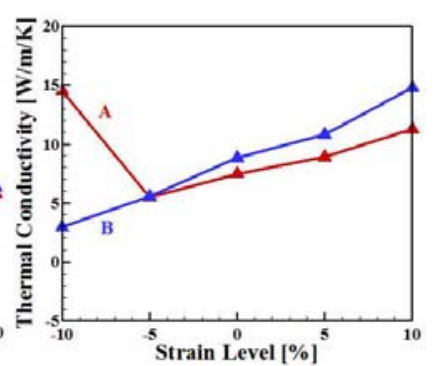

(c)

Figures 9 (a) to 9 (c) displays the thermal properties of the examined TC-HAP nanocomposite material systems (NS: with needle shaped HAP and PS: with plate shaped HAP). Thermal diffusivity shows an increasing trend, in general, with increase in strain levels. The specific heat and thermal conductivity values of NS nanocomposite increase when applying compressive strain, while those decrease in the case of the PS nanocomposite, Figures 9(b) and 9(c). Effect of tensile straining is to increase both properties. Applying compressive straining (and stress) would increase the bonded energy (e.g., stretching energy, bending energy et al.) at the examined compressive strain levels. The non-bonded energy comes from the van der Waals and electrostatic forces, which is shown in equation (10) and (11). Applying compressive strain leads to the reduction in interatomic distance $r_{i j}$, which will increase the non-bonded energy $\left(E_{V D W}\right.$ and $\left.E_{\text {electrostatic }}\right)$.

On the contrary, applying the tensile strain leads to the increasing of the interatomic distance $r_{i j}$, which leads to the decreasing of the non-bonded energy $\left(E_{V D W}\right.$ and $E_{\text {electrostatic }}$ ). These factors contribute to increase in energy required to induce thermal motion while compressing and reduction in energy required to induce thermal motion while in tension for the PS composite. Increasing interface area in the case of NS composite negates the influence of non-bonded interactions. Therefore, there is an increase in specific heat during compression as well as during tension.

$$
E_{V D W}=\sum_{i}^{N} \sum_{j}^{N}\left[\left(\frac{A_{i j}}{r_{i j}}\right)^{12}-\left(\frac{B_{i j}}{r_{i j}}\right)^{6}\right] w_{i j}
$$




$$
E_{\text {electrostatic }}=\sum_{i}^{N} \sum_{j}^{N} \frac{q_{i} q_{j}}{r_{i j}} w_{i j}
$$

Thermal conductivity has been shown to be dependent on the strain as $k \sim T^{-1} \varepsilon^{-\gamma}$, where, $\gamma$ is a material constant, $\varepsilon$ the strain values and $T$ gives the temperature (Bhowmick and Shenoy, 2006). This leads to the increase of the thermal conductivity when applying compressive or tensile strain, which agrees with the observed trend in the variation of thermal conductivity of the nanocomposite with needle shaped HAP minerals as a function of strain [red curve Figure 9(c)]. In order to explain the variation of thermal conductivity with straining, we can rewrite equation (9) as (Bhowmick and Shenoy, 2006; Samvedi and Tomar, 2010).

$$
k=\frac{1}{3} C v^{2} \tau_{p h} \quad \text { or } \quad k=\frac{1}{3} C r_{i j}^{2} \frac{g}{m} \tau_{p h} .
$$

Here $\tau_{p h}$ gives phonon-phonon interaction or phonon relaxation time, $g$ represents the stiffness constant of the atomic structure and $m$ gives the mass of atoms. In the above equation, the relations $l=v \tau_{p h}$ and $v=r_{i j} \sqrt{g / m}$ are used. Here, $r_{i j}$ represents the average interatomic distance. From equation (12) it can be shown that an increase in the compression causes a decrease in $r_{i j}$ and hence a decrease in phonon relaxation time. In addition, it leads to an increase in the stiffness constant and, therefore to lattice an harmonicity (Samvedi and Tomar, 2009). However, the effect of increase in stiffness may be lower than the effect of decrease in interatomic distance and phonon relaxation time. Hence, we see a decrease in thermal conductivity with an increase in compressive strain. Effect of such factors is minimised in high interface area NS nanocomposite structure.

Overall, the difference between the thermal properties (specific heat and thermal conductivity) variation as a function of strain could be attributed to the shape effect of the mineral parts (HAP) but the model to explain such behaviour is not available. What is interesting to observe is that trend in the change of thermal properties can be tailored as a function of strain as well as interfacial arrangements. Models are needed that take into account the influence of varied structural arrangement and shape effect of the component in nanocomposites mimicking the biological materials. The focus of the present work is on analysing this behaviour using classical molecular simulations. Development of classical models to explain such behaviour is undergoing.

\section{Conclusions}

The present study developed a simulation tool to calculate the thermal properties of TC-HAP interfacial biomaterial system and analysed thermal and mechanical properties of a hierarchy of interfaces. From the analyses of the results, the following conclusions can be drawn.

1 There is significant size effect on the thermal diffusivity and thermal conductivity of the TC-HAP material systems. The rate of increase of thermal conductivity is found to be higher than that of the thermal diffusivity. Thermal diffusivity of the TC-HAP interfacial systems is found to increase with increase in the number of interfaces periods. 
2 The thermal properties of the HAP system show less variation as a function of strain than those of the TC molecules, which is attributed to the difference between the crystalline structure of the HAP and the polymer chain structure of the TC. The TC-HAP interfacial system is found to have thermal properties in between those of TC and HAP. The interface introduction also leads to increase in strength. Strain is observed to be an important factor which can prove significant in tailoring the thermal properties of TC-HAP systems.

3 Thermal properties of TC-HAP nanocomposite with different shape of minerals (HAP) were observed to have different response to the applied strain. Applying either compressive or tensile strain results in increasing in thermal conductivity of the nanocomposite with needle shaped HAP. However, in the case of plated shaped HAP, compressive strain leads to the reduction in thermal conductivity as well as specific heat while tensile strain leads to the increase in these properties. Such difference was attributed to the change in interfacial arrangement. However, more accurate analytical models to explain such behaviour with an account of the factors such as the interface arrangement and shape effect of the component are needed.

4 The thermal diffusivity values show variation as a function of strain. By applying tensile or compressive strain to the material system the proportional or inversely proportional trend can be tailored.

\section{References}

Algaer, E.A. and Müller-Plathe, F. (2011) 'Molecular dynamics calculations of the thermal conductivity of molecular liquids, polymers and carbon nanotubes', Soft Materials, Vol. 10, Nos. 1-3, pp.42-80.

Anderson, D. (2005) Collagen Self-Assembly: A Complementary Experimental and Theoretical Perspective, PhD Dissertation, University of Toronto.

Autumn, K., Liang, Y.A. et al. (2000) 'Adhesive force of a single gecko foot-hair', Nature, Vol. 405, No. 6787, pp.681-685.

Bella, J., Eaton, M. et al. (1994) 'Crystal and molecular structure of a collagen-like peptide at 1.9 angstrom resolution', Science, Vol. 266, No. 5182, pp.75-81.

Bhowmick, S. and Shenoy, V.B. (2006) 'Effect of strain on the thermal conductivity of solids', $J$ Chem Phys, Vol. 125, No. 16, p.164513.

Bhowmik, R., Katti, K.S. et al., (2007) 'Molecular dynamics simulation of hydroxyapatitepolyacrylic acid interface', Polymer, Vol. 48, No. 2, pp.664-674.

Biyikli, S., Modest, M.F. et al. (1986) 'Measurements of thermal properties for human femora', Journal of Biomedical Materials Research, Vol. 20, No. 9, pp.1335-1345.

Boonrungsiman, S., Gentleman, E. et al. (2012) 'The role of intracellular calcium phosphate in osteoblast-mediated bone apatite formation', Proceedings of the National Academy of Sciences, Vol. 109, No. 35, pp.14170-14175.

Buehler, M.J. (2006a) 'Atomistic and continuum modeling of mechanical properties of collagen: elasticity, fracture, and self-assembly', Journal of Materials Research, Vol. 21, No. 8, pp.1947-1962.

Buehler, M.J. (2006b) 'Nature designs tough collagen: explaining the nanostructure of collagen fibrils', Proceedings of the National Academy of Sciences of the United States of America, Vol. 103, No. 33, No. 2, pp.12285-12290. 
Cottin, D., Shillito, B. et al. (2010) 'Identification of differentially expressed genes in the hydrothermal vent shrimp $<\mathrm{i}>$ Rimicaris exoculata $</ \mathrm{i}>$ exposed to heat stress', Marine Genomics, Vol. 3, No. 2, pp.71-78.

Dubey, D.K. and Tomar, V. (2009) 'Role of the nanoscale interfacial arrangement in mechanical strength of tropocollagen-hydroxyapatite based hard biomaterials', Acta Biomaterialia, Vol. 5, No. 7, pp.2704-2716.

Dubey, D.K. and Tomar, V. (2010) 'Role of molecular level interfacial forces in hard biomaterial mechanics: a review', Annals of Biomedical Engineering, Vol. 38, No. 6, pp.2040-2055.

Elliott, J.C. Mackie, P.E. et al. (1973) 'Monoclinic hydroxyapetite', Science, Vol. 8, No. 4090, pp.1055-1057.

Eppell, S.J., Smith, B.N. et al. (2005) 'Nano measurements with micro-devices: mechanical properties of hydrated collagen fibrils', Journal of the Royal Society Interface, Vol. 3, No. 6, pp.117-121.

Fratzl, P., Fratzlzelman, N. et al. (1991) 'Nucleation and growth of mineral crystals in bone studied by small-angle X-ray scattering’, Calcified Tissue International, Vol. 48, No. 6, pp.407-413.

Gao, H. (2006) 'Application of fracture mechanics concepts to hierarchical biomechanics of bone and bone-like materials', International Journal of Fracture, Vol. 138, Nos. 1-4, pp.101-137.

Gao, H.J. and Wang, X. (2005) 'Mechanics of hierarchical adhesion structures of geckos', Mechanics of Materials, Vol. 37, Nos. 2-3, pp.275-285.

Gao, H.J., Ji, B.H. et al. (2003) 'Materials become insensitive to flaws at nanoscale: lessons from nature', Proceedings of the National Academy of Sciences of the United States of America, Vol. 100, No. 10, pp.5597-5600.

Gupta, H.S., Seto, J. et al. (2006a) 'Cooperative deformation of mineral and collagen in bone at the nanoscale', Proceedings of the National Academy of Sciences of the United States of America, Vol. 103, No. 47, pp.17741-17746.

Gupta, H.S., Wagermaier, W. et al. (2006b) 'Fibrillar level fracture in bone beyond the yield point', International Journal of Fracture, Vol. 139, Nos. 3-4, pp.425-436.

Handgraaf, J.W. and Zerbetto, F. (2006) 'Molecular dynamics study of onset of water gelation around the collagen triple helix', Proteins: Structure, Function, and Bioinformatics, Vol. 64, No. 3, pp.711-718.

Hauptmann, S., Dufner, H. et al. (2003) 'Potential energy function for apatites', Physical Chemistry Chemical Physics, Vol. 5, No. 3, pp.635-639.

Huang, C.C., Couch, G.C. et al. (1998) 'The object technology framework (OTF): an object-oriented interface to molecular data and its application to collagen', Pacific Symposium on Biocomputing, pp.349-360.

Huang, X., Huai, X. et al. (2009) 'Thermal transport in Si/Ge nanocomposites', J. Phys. D: Appl. Phys., Vol. 42, No. 9, p.095416.

Huiskes, R. (1980) 'Some fundamental aspects of human joint replacement, section II: heat generation and conduction analyses of acrylic bone cement in situ', Acta Orthop. Scan., Suppl, Vol. 185, p.43.

Isgro, T., Phillips, J. et al. (2012) 'Analysis, specific heat', NAMD Tutorial, pp.50-53.

Jager, I. and Fratzl, P. (2000) 'Mineralized collagen fibrils: a mechanical model with a staggered arrangement of mineral particles', Biophysical Journal, Vol. 79, No. 4, pp.1737-1746.

Jeng, M-S., Yang, R. et al. (2008) 'Modeling the thermal conductivity and phonon transport in nanoparticle composites using Monte Carlo simulation', Journal of Heat Transfer, Vol. 130, No. 4, pp.022410-022411.

Ji, B.H. (2008) 'A study of the interface strength between protein and mineral in biological materials', Journal of Biomechanics, Vol. 41, No. 2, pp.259-266.

Klein, T.E. and Huang, C.C. (1999) 'Computational investigations of structural changes resulting from point mutations in a collagen-like peptide', Biopolymers, Vol. 49, No. 2, pp.167-183. 
Kramer, R.Z., Venugopal, M.G. et al. (2000) 'Staggered molecular packing in crystals of a collagen-like peptide with a single charged pair', Journal of Molecular Biology, Vol. 301, No. 5, pp.1191-1205.

Landis, W.J., Hodgens, J.J. et al. (1996a) 'Structural relations between collagen and mineral in bone as determined by high voltage electron microscopic tomography', Microscopy Research and Technique, Vol. 33, No. 2, pp.192-202.

Landis, W.J., Hodgens, K.J. et al. (1996b) 'Mineralization of collagen may occur on fibril surfaces: evidence from conventional and high-voltage electron microscopy and three-dimensional imaging', Journal of Structural Biology, Vol. 117, No. 1, pp.24-35.

Lorenzo, A.C. and Caffarena, E.R. (2005) 'Elastic properties, Young's modulus determination and structural stability of the tropocollagen molecule: a computational study by steered molecular dynamics', Journal of Biomechanics, Vol. 38, No. 7, pp.1527-1533.

Moses, W.M., Witthaus, F.W. et al. (1995) 'Measurement of the thermal conductivity of cortical bone by an inverse technique', Experimental Thermal and Fluid Science, Vol. 11, No. 1, pp.34-39.

Nadareishvili, M., Burjanadze, T., Kvavadze, K., Kekelidze, N., Kiziria, E. and Gelagutashvili, E. (2012) Pulsed Differential Scanning Calorimeter Measurement of Collagene Fiber Heat Capacity, arXiv: 1206.3908.

Nelson, J.S., Yow, L. et al. (1988) 'Ablation of bone and methacrylate by a prototype mid-infrared erbium: YAG laser', Lasers in Surgery and Medicine, Vol. 8, No. 5, pp.494-500.

Orgel, J., Irving, T.C. et al. (2006) 'Microfibrillar structure of type I collagen in situ', Proceedings of the National Academy of Sciences of the United States of America, Vol. 103, No. 24, pp.9001-9005.

Phillips, J.C., Braun, R. et al. (2005) 'Scalable molecular dynamics with NAMD', Journal of Computational Chemistry, Vol. 26, No. 16, pp.1781-1802.

Ponder, J.W. and Case, D.A. (2003) 'Force fields for protein simulations', Protein Simulations, Vol. 66, pp.27-85, Academic Press Inc., San Diego.

Qu, T. and Tomar, V. (2014) 'An analysis of the effects of temperature and structural arrangements on the thermal conductivity and thermal diffusivity of tropocollagen-hydroxyapatite interfaces', Materials Science and Engineering, Vol. C 38, No. 1, pp.28-38.

Radmer, R.J. and Klein, T.E. (2004) 'Severity of osteogenesis imperfecta and structure of a collagen-like peptide modeling a lethal mutation site', Biochemistry, Vol. 43, No. 18, pp.5314-5323.

Samvedi, V. and Tomar, V. (2009) 'Role of heat flow direction, monolayer film thickness, and periodicity in controlling thermal conductivity of a Si-Ge superlattice system', Journal of Applied Physics, Vol. 105, No. 1, pp.013541-013549.

Samvedi, V. and Tomar, V. (2010) 'The role of straining and morphology in thermal conductivity of a set of Si-Ge superlattices and biomimetic Si-Ge nanocomposites', Journal of Physics D: Applied Physics, Vol. 43, No. 13, p.135401.

Samvedi, V. and Tomar, V. (2011) 'Correlation of thermal conduction properties with mechanical deformation characteristics of a set of SiC-Si[sub 3] $[$ [sub 4] nanocomposites', Journal of Engineering Materials and Technology, Vol. 133, No. 1, p.011013.

Sasaki, N. and Enyo, A. (1995) 'Viscoelastic properties of bone as a function of water-content', Journal of Biomechanics, Vol. 28, No. 7, pp.809-815.

Sasaki, N. and Odajima, S. (1996a) 'Elongation mechanism of collagen fibrils and force-strain relations of tendon at each level of structural hierarchy', Journal of Biomechanics, Vol. 29, No. 9, pp.1131-1136.

Sasaki, N. and Odajima, S. (1996b) 'Stress-strain curve and Young's modulus of a collagen molecule as determined by the X-ray diffraction technique', Journal of Biomechanics, Vol. 29, No. 5, pp.655-658.

Schelling, P.K., Phillpot, S.R. et al. (2002) 'Comparison of atomic-level simulation methods for computing thermal conductivity', Physical Review B, Vol. 65, No. 144306, pp.1-12. 
Tiktopulo, E.I. and Kajava, A.V. (1998) 'Denaturation of type I collagen fibrils is an endothermic process accompanied by a noticeable change in the partial heat capacity', Biochemistry, Vol. 37, No. 22, pp.8147-8152.

Vesentini, S., Fitie, C.F.C. et al. (2005) 'Molecular assessment of the elastic properties of collagen-like homotrimer sequences', Biomechanics and Modeling in Mechanobiology, Vol. 3, No. 4, pp.224-234.

Wallace, J.M. (2012) 'Applications of atomic force microscopy for the assessment of nanoscale morphological and mechanical properties of bone', Bone, Vol. 50, No. 1, pp.420-427.

Weiner, S., Talmon, Y. et al. (1983) 'Electron diffraction of mollusc shell organic matrices and their relationship to the mineral phase', International Journal of Biological Macromolecules, Vol. 5, No. 6, pp.325-328.

Wiggins, K. and Malkin, S. (1976) 'Drilling of bone', Journal of Biomechanics, Vol. 9, No. 9, pp.553-559.

Yang, R. and Chen, G. (2004) 'Thermal conductivity modeling of periodic two-dimensional nanocomposites', Phys. Rev., Vol. 69, No. 19, pp.5316-5326.

Yang, R., Chen, G. et al. (2005) 'Thermal conductivity of simple and tubular nanowire composites in the longitudinal direction', Phys. Rev., Vol. 72, No. 12, pp.5418-5425.

Zelenov, E.S. (1986) 'Experimental investigation of the thermophysical properties of compact bone', Mechanics of Composite Materials, Vol. 21, No. 6, pp.759-762.

Zhang, D., Chippada, U. et al. (2007) 'Effect of the structural water on the mechanical properties of collagen-like microfibrils: a molecular dynamics study', Annals of Biomedical Engineering, Vol. 35, No. 7, pp.1216-1230. 